\title{
Silicon Carbide detectors: a concrete perspective for nuclear-astrophysics experiments
}

\author{
Salvatore Tudisco ${ }^{1, *}$ for SiCILIA Collaboration \\ ${ }^{1}$ INFN-LNS via S. Sofia 62, 95123 Catania, Italy
}

\begin{abstract}
Silicon Carbide, also known as carborundum, is one of the most interesting materials for the production of detection systems. This contribution presents an overview of the results of SiCILIA activities, an R\&D totally funded by INFN. Detector manufacturing and the general performance are discussed.
\end{abstract}

\section{Introduction}

Today, the frontier of experimental activities of nuclear and particles physics requires compact detectors with good energy and the ability to operate at high fluxes (up to $10^{14} \mathrm{~cm}^{2}$ ) and high rate, in order to measure cross sections of very rare phenomena [1-4]. Silicon Carbide (SiC) represent the new challenge in the detectors technology for its excellent performance in terms of radiation hardness.

Due to its composition $\mathrm{SiC}$ is a wide indirect band gap semiconductor and it is the only stable compound in the binary phase diagram of the two groups IV elements, silicon and carbon. Of all the wide band gap semiconductors, silicon carbide is presently the most intensively studied one and the one with the highest potential to reach market maturity in a wide field of device applications, such as high-temperature electronics, biomedical sensors [5], UV photo-sensors [6], particle and X-ray detectors.

$\mathrm{SiC}$ is also seriously considered as a valid alternative to silicon for the production of radiation hard devices, since it gives the opportunity to couple the excellent properties of silicon detectors (efficiency, linearity, resolution) with a much larger radiation hardness, thermal stability and insensitivity to visible light.

$\mathrm{SiC}$ can occur in various types of crystal structures according to the stacking sequence of the atoms in the lattice, this property is known with the name of "polytypism". SiC exists in more than 200 different polytypes; the $3 \mathrm{C}, 4 \mathrm{H}$ and $6 \mathrm{H}$ structures are the most common and popular for microelectronics applications. Each polytype has its own physical properties such as the energy bandgap, ranging from $2.36 \mathrm{eV}$ in $3 \mathrm{C}$ to $3.23 \mathrm{eV}$ in $4 \mathrm{H}$. The $4 \mathrm{H}-\mathrm{SiC}$ is considered to be the most appropriate for high-power, high-frequency, and high-temperature applications.

Low defect material used for device application is typically obtained growing epitaxial layers by a CVD (Chemical Vapour Deposition) technique. Epitaxy allows also the highly

* Corresponding author: tudisco@lns.infn.it 
precise control of thickness, doping and homogeneity of crystal layers. The growth of $4 \mathrm{H}-$ $\mathrm{SiC}$ polytype requires temperatures ranging from 1600 to $1650^{\circ} \mathrm{C}$ and during the $\mathrm{CVD}$ process the dopants can also provided by means of gaseous precursors. Epitaxy is the fundamental ingredient for the construction of a radiation detector.

\section{Detectors manufacturing}

A junction, operating under reverse bias, is a typical structure used for the construction of a particle detectors. In order to avoid a reduction in the leakage current, a lowest concentration of impurities and defects is required. The wide $\mathrm{SiC}$ band-gap reduces significantly the rate of thermally generated charge carriers, reducing the leakage current and the noise level. On the other hand, this could also represent a disadvantage, because for a particle with a defined energy which is ideally detected the amount of charge carriers generated are about twice in $\mathrm{Si}$ than in $\mathrm{SiC}$. Therefore, detectors based on $\mathrm{SiC}$ have lower pulse amplitudes. However, for small signals, the reduction of the noise level overcompensates the reduction of the signal level, so that the overall Signal-to-Noise Ratio (SNR) is improved for SiC detectors. Furthermore, SiC detectors still have a high SNR at higher temperatures with respect to Si devices unless external cooling is used to decrease the intrinsic carrier level.

Thicknesses, dimensions (detection area) and manufacture technologies are the actual technological limits on $\mathrm{SiC}$ material processing. Most of the commercial SiC devices are realized through Schottky junctions implementation. In view of the potential application of $\mathrm{SiC}$ as radiation hard material for detectors implementation and thanks to the request for several new and ambitious projects of Italian National Institute of Nuclear Physics (INFN) in fundamental and applied nuclear physics (NUMEN [7], NuReLP [8-12], ELIMED $[13,14])$ a cooperation has been started between INFN and IMM-CNR for a common R\&D activity on SiC technology. A specific project, named SiCILIA (Silicon Carbide detectors for Intense Luminosity Investigations and Applications) [15], has been totally funded by INFN. Some of the most important results concerning the technological issues and the particle detection applications has been recently published [16-18].

The first step for the construction of SiC detectors is the growths of an epitaxial layers on a substrate; these layers are considered as the active thicknesses. In the second step the layers are used for the detectors implementation. To obtain a high-quality material, one uses as seeds substrate with the lowest possible defect concentration and an epitaxial process able to reduce the density of defects present in the substrates. Some growth parameters, such as the growth rate condition, have a strong influence on the defect formation or annihilation. These parameters are then crucial to obtain large area and thick devices.

Second step of the processing is the epitaxial grow of thin double layer necessary for the formation of $\mathrm{p}^{+} / \mathrm{n}$ junction. A $\mathrm{p}^{+}$layer $0.3-0.5 \mu \mathrm{m}$ thick with a doping concentration of the order of $10^{18}-10^{19} / \mathrm{cm}^{3}$ was grown over the n-epi-layer with a doping concentration in the range $5-8 \cdot 10^{13} / \mathrm{cm}^{3}$ ). After this step, a photolithography for the definition of the detector area by a ICP plasma etching was performed. Then, a second photolithography was performed for the implementation of the edge structures to reduce the electric field at the device borders. In particular these edge structures were performed by using a metal field plate.

The process continues with the deposition of an isolation oxide and the opening of the contacts with a further photolithographic process. Then the front metallization (Ni) has been deposited, defined with a further photolithographic process and subsequently annealed to form a good ohmic contact on the $\mathrm{p}^{+}$region. Only on the periphery of the detector a thicker layer of $\mathrm{Ti}$ and $\mathrm{Al}$ was deposited for the bonding. Finally, the ohmic contact was formed by a titanium/nickel/gold deposition [15]. 


\section{Detectors performance}

At the end of the processing we have got devices of several dimension and thickness. To investigate the devices performance several measurements in different configurations (on test bench or in operative conditions) were conducted. By using, for example, the alpha particles of radioactive source $\left({ }^{239} \mathrm{Pu},{ }^{241} \mathrm{Am},{ }^{244} \mathrm{Cm}\right)$ and a standard spectroscopic electronics chain we obtained an energy resolution of about $0.4 \%(42.8 \pm 1.1 \mathrm{keV})$ for alphas coming from ${ }^{241} \mathrm{Am}(5.486 \mathrm{MeV})$ while a commercial Hamamatsu silicon detector (S3590-06) gives under the same conditions about $0.22 \%(21.4 \pm 0.8 \mathrm{keV})$ [15]. Such difference is partially due to differences in: $i$ ) the dead layers of entrance zone of the detectors; $i i)$ the energy of e-h pair creation; $i i i)$ the fluctuations of charge collection; $i v$ ) the electronic noise contribution.

The response function of $\mathrm{SiC}$ detector to the energetic neutrons, in comparison with Single Cristal Diamond (SCD) detector, ware investigated by irradiating the devices with 14.1 MeV neutrons at the ENEA-Frascati Neutron Generator facility. In this facility, neutrons are produced by Deuterium-Tritium (DT) reactions obtained from deuterium ions accelerated up to $300 \mathrm{keV}$ impinging on a tritiated-titanium target. A dedicated custom electronic chain was used to bias and collect charge carriers from each detector. In particular, the SCD were coupled to a fast charge preamplifier while the $\mathrm{SiC}$ devices was pre-amplified by a spectroscopic amplifier producing a Gaussian output; both signals were directly fed into a digitizer [18]. The Pulse Height Spectrum (PHS) of SiC detectors shows a more complicated structure in respect to that of SCD, this is due to the presence of the Si atoms and in particular to the neutron interaction via $(\mathrm{n}, \alpha)$ and $(\mathrm{n}, \mathrm{p})$ reactions. This complexity limits the sensitivity of the $\mathrm{SiC}$ when it is used as a neutron spectrometer for Deuterium-Tritium plasma diagnostics $[10,11]$, though it could be well suited to measure the temperature in thermal plasmas. On the other hand, the detectors showed a good efficiency and the absence of instabilities during neutron irradiation up to a $14 \mathrm{MeV}$ neutron fluence of $4.45 \cdot 10^{11} \mathrm{n} / \mathrm{cm}^{2}$ suggests a straightforward use of this detector as a fast neutron diagnostic. Furthermore, it could be successfully used as a neutron diagnostic in those environments in which small size is a requirement, such as in a neutron camera. In addition, the possibility of growing Silicon Carbide layers with different thicknesses allows for tuning the neutron detection efficiency, and, therefore, using $\mathrm{SiC}$ crystals as charged particle detectors in those environments where high neutron fluxes are an issue, such as in FILD detectors.

\subsection{Radiation Hardness}

As mentioned in the previous sections $\mathrm{SiC}$ are very robust devices; they are able to sustain very high neutron fluxes, remaining stable.

Radiation damage of our SiC detectors has been investigated by irradiating the devices with an $\mathrm{H}^{+}$beam at $60 \mathrm{MeV} / \mathrm{A}$ delivered by the Superconducting cyclotron of INFN-LNS (Laboratori Nazionali del Sud) facility. The total dose delivered on the devices was $3 \mathrm{kGy}$, and no significant changes in electrical characteristics, energy resolution and charge collection were observed.

In order to establish the damage threshold, a further study has been conducted, irradiating some devices with a heavy ions beam delivered by the Tandem accelerator of INFN-LNS Facility. In these experiments Oxygen ions was implanted inside active thickness of the detector and the overall performance of the devices (stability, resolution, charge collection) has been monitored. The results of these studies demonstrate how SiC detectors are able to withstand high fluences of implanted ions, up to about $10^{13}$ ions $/ \mathrm{cm}^{2}$. The devices do not show serious damage to these fluences; only a decrease in the charge collection efficiency, at around $40 \%$. This effect is generated by the creation of huge number of defects near the ion stopping point, within the material. 

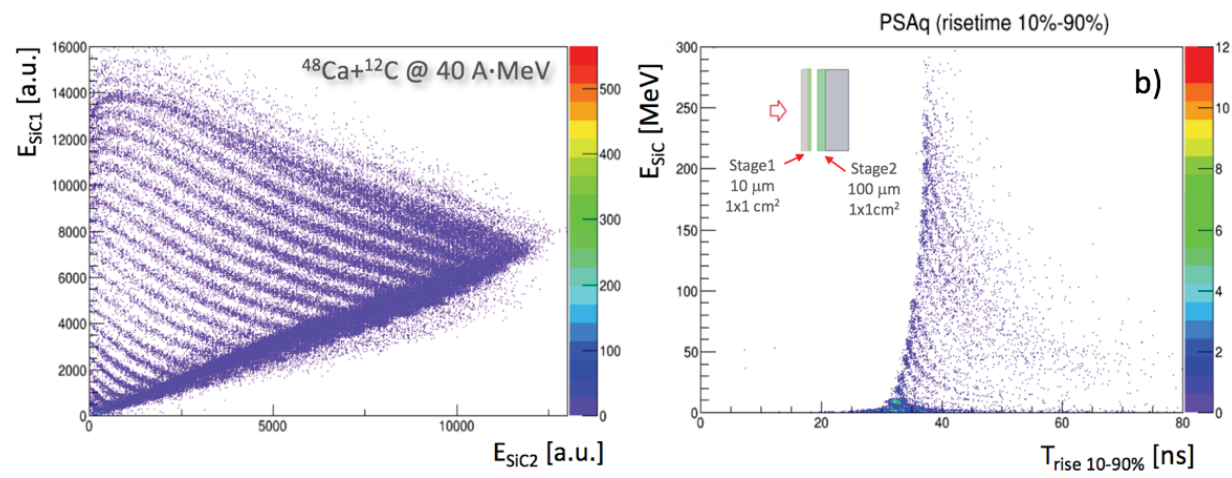

Fig. 1. a) $\Delta \mathrm{E}-\mathrm{E}$ correlation obtained from the first two stages of telescope ( $\mathrm{SiC} 1$ and $\mathrm{SiC} 2)$; b) Pulse shape identification for fragments stopped in $\mathrm{SiCl}$ (stage 1) Deposited energy vs Rise Time of the charge signal.

\subsection{Particles Identification}

Some of $\mathrm{SiC}$ devices were mounted in telescope configuration and used to detect nuclear fragments, in order to test their identification capabilities both with the $\Delta \mathrm{E}-\mathrm{E}$ and the Pulse Shape Analysis (PSA) techniques [16]. The $\Delta \mathrm{E}-\mathrm{E}$ technique can be used for particles with kinetic energy large enough to pass through the first detector and low enough to be stopped in the second. The partition of the energy between the two detectors is different for different particles, due to their different stopping power. In PSA, only particles stopped in the first detector (i.e. those that cannot be identified with the $\Delta \mathrm{E}-\mathrm{E}$ technique) are considered. In fact, different nuclear fragments having the same kinetic energy have a different energy deposition "profile" as a function of the penetration depth, thus producing different charge collection times and, consequently, different pulse shapes. The aim of the measurement was to verify the performance of $\mathrm{SiC}$, by detecting and identifying heavy nuclear fragments produced over a large range of charges, masses and energies, like the one we expect in an actual physics experiment. The measurement was conducted in the "Ciclope" scattering chamber at the INFN-LNS in Catania. Beam of ${ }^{48} \mathrm{Ca}$ at $40 \mathrm{~A} \cdot \mathrm{MeV}$ from Superconducting cyclotron were used to produce nuclear collisions on a thin ${ }^{12} \mathrm{C}$ target. Telescope were mounted in the scattering chamber, at distances of about $80 \mathrm{~cm}$ from the target. They were positioned at a polar angle of about $8^{\circ}$, i.e. as close to the grazing angle. The detectors are read-out by hybrid charge preamplifiers and the output of is acquired by a dedicated channel of a custom digitizing board, featuring 14-bit 125 MSPS sampling ADCs. In order to avoid dead layers between the first and the second stage of the telescope, the first stage is reverse mounted while the second is front mounted. Fig. 1a shows the $\Delta \mathrm{E}-\mathrm{E}$ correlation obtained from the two stages of telescope where the different ridges correspond to different atomic numbers of the impinging fragments. The $100 \mu \mathrm{m}$ thick dead layer in front of the first active layer does not affect its identification properties, only reducing the energy of the fragments before they enter the active region. Elements up to $\mathrm{Z}=22$ are well resolved. Fig. $1 \mathrm{~b}$ we report the correlation of the deposited energy, $\mathrm{E}$ vs the rise-time ( $10 \%$ to $90 \%$ of the maximum value) of the charge signal. The ridges associated to the different elements can be clearly identified.

\section{Conclusions}


The results of the SiCILIA collaboration clearly demonstrate how Silicon Carbide represent today a valid alternative to Silicon for the production of radiation hard detectors. They exhibit excellent performance in terms of energy resolution, comparable to highperformance commercial devices. Can be used for the detection and identification of charged particles when are mounted in a telescopic configuration. Moreover, through the pulse shape analysis it is possible to identify the charge particles stopped inside their active thickness. $\mathrm{SiC}$ devices can also be used for neutron detection or monitoring; they have some limits when are used as a high energy neutrons spectrometer, but on the other hand showed a good efficiency and the absence of instabilities. They could be well suited to measure the temperature in thermal plasmas or can be used to detect ions, electrons, energetic particles, $\mathrm{X}$-ray photons in a laser produced plasmas [19].

Currently, the study for possible applications of these devices as dosimeters is being completed. Silicon carbide can host point defects in the crystal lattice which are known as color centers. These defects can generate single photons on demand and thus serve as a platform for single-photon source. Such devices could be a fundamental resource for many emerging applications of quantum information science.

\section{References}

1. A. Muoio et al. EPJ Web of Conferences 117, 10006 (2016)

2. C. Spitaleri et al. Phys. Rev. 95, Issue:3, 035801 (2017)

3. M. La Cognata et al. Phys. Lett. B 664, Issue:3, 157 (2008)

4. A. Tumino et al. Phys. Lett. B 750, 59 (2015)

5. A. Scordino et al. Jour. of Phys. D 41, Issue:15, 155507 (2008)

6. S. Privitera et al Sensors 8, Issue:8, 4636 (2008)

7. F. Cappuzzello et al. Eurp. Phys. Jour. A 54, Issue:5, 72 (2018)

8. F. Negoita et al. Romania Reports in Phys. 68, S37 (2016)

9. D. Mascali et al. Rad. Eff. And Defec. in Solids 165, Issue:6, 730 (2010)

10. G. Cristoforetti et al. Plasma Phys. And Contr. Fus. 56, Issue:9, 095001 (2014)

11. D. Mascali et al. Eur. Phys. Lett. 100, Issue:4, 45003 (2012)

12. N. Gambino et al. Appl. Surf. Scie. 272, 69 (2013)

13. F. Schillaci et al. Jour. of Phys. Conf. 508, Issue:1, 012010 (2014)

14. G.A.P. Cirrone et al. Proc. of SPIE 8779, 87791 (2013)

15. S. Tudisco et al. Sensors 18, Issue:7, 2289 (2018)

16. C. Ciampi et al. Nucl. Instr. \& Meth. A 925, 60 (2019)

17. S. Nida et al. Jour. of Synch Rad. 26, 28 (2019)

18. M. Rebai et al Nucl. Instr. \& Meth. A 946, 162637 (2019)

19. S. Tudisco et. al. Nucl. Instr. \& Meth. A 653, Issue:1, 47 (2011) 